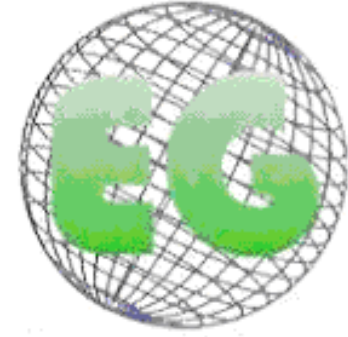

ISSN 1695-6141

\title{
La importancia del apoyo socio-emocional en adolescentes y adultos jóvenes portadores de enfermedad crónica: una revisión de literatura
}

A importância do apoio sócio-emocional em adolescentes e adultos jovens portadores de doença crônica: uma revisão de literatura

The importance of social-emotional support in adolescents and young adults with chronic disease: a literature review

\section{*Schütz Balistieri, Aline ** Mara de Melo Tavares, Claúdia}

\begin{abstract}
*Mestranda en Ciencias del Cuidado en Salud por la Universidad Federal Fluminense. Enfermera Supervisora de la Comisión de Control de Infección Hospitalaria del Hospital São João Batista, Volta Redonda, Rio de Janeiro. E-mail: line-ac@ig.com.br $\quad{ }^{* *}$ Doctora en Enfermería por la Universidad Federal de Rio de Janeiro y pos-doctorado por la USP-SP. Profesora Titular de la Universidad Federal Fluminense. Brasil.
\end{abstract}

Palabras clave: apoyo social; adulto joven; adolescente; enfermedad crônica

Palavras-chave apoio social; adulto jovem; adolescente; doença crônica

Keywords: social support; young adult; adolescent and chronic disease

\section{RESUMEN}

Este estudio cualitativo tuvo como objetivo identificar la importancia del apoyo emocional y social en adolescentes y adultos jóvenes con enfermedades crónicas y la importancia del equipo de salud, en especial las enfermeras, en la prestación de apoyo emocional y social. La encuesta se realizó mediante la búsqueda de artículos en revistas, bases de datos electrónicas de la Biblioteca Virtual en Salud, Scielo, Lilacs y BDENF. El apoyo socio-emocional se convierte en casi imprescindible para ayudar a estos jóvenes a tener el comportamiento más adecuado posible en la enfermedad que los acompaña, y para tener una buena lucha contra la enfermedad. Es importante que los profesionales de la salud incorporen valores de apoyo emocional en el plan de atención, porque es de esto que podemos ofrecer una vida mejor con los sentimientos y situaciones que les acompañarán y, por consiguiente, pueden llevar a cabo el tratamiento propuesto de mejor manera posible. Por lo tanto, se concluye que el apoyo socio-emocional es fundamental para estos pacientes que pueden vivir con la enfermedad de la manera más adecuada, que debe entenderse por profesionales de la salud como un acto de atención que se debe insertar en sus actividades con el paciente .

\section{RESUMO}

Estudo qualitativo que objetivou identificar a importância do apoio sócio-emocional em adolescentes e adultos jovens portadores de doença crônica e a importância da equipe de saúde, em especial o enfermeiro, no fornecimento do apoio sócio-emocional. O levantamento de dados foi realizado através 
da busca de artigos nos periódicos da área, nas bases eletrônicas de dados da Biblioteca Virtual em Saúde, SCIELO, LILACS e BDENF. O apoio sócio-emocional torna-se praticamente fundamental no auxílio desses jovens, para que os mesmos tenham o comportamento mais adequado possível dentro da enfermidade que os acompanha e para que haja um bom enfrentamento da doença. É importante que os profissionais de saúde incorporem o apoio sócio-emocional no plano de cuidados, pois é a partir dele que poderemos proporcionar uma melhor convivência com os sentimentos e situações que irão os acompanhar e, consequentemente, os mesmos poderão realizar o tratamento proposto da melhor forma possível. Desse modo, concluímos que o apoio sócio-emocional é fundamental para que esses pacientes possam conviver com a doença da forma mais adequada possível, devendo ser entendido pelos profissionais de saúde como um ato de cuidado que deve estar inserido dentro de suas atividades com o paciente.

\section{ABSTRACT}

It is a qualitative study aimed to identify the importance of socio-emotional support in adolescents and young adult patients with chronic disease and the importance of the health team, especially the nurses, in the provision of socio-emotional support. The data collection was done through the search for articles in journals of the area, in the electronic database of the Virtual Health Library, SCIELO, LILACS and BDENF. The socio-emotional support becomes practically essential in helping these young people to have the behavior more appropriate as possible within the disease that accompanies them and have a good dealing with the disease. It's important that health professionals include the socio-emotional support in the care plan, because it's from this that we would be able to provide a better sociability with the feelings and situations that will accompany them and, consequently, they may carry out the proposed treatment in the best possible way. In this way, we conclude that the socio-emotional support is essential to these patients to live with the disease in the most appropriate way, and it should be understood by health professionals as an act of care that must be added into its activities with the patient.

\section{INTRODUCCIÓN}

La adolescencia es un período de la vida en el que surgen dificultades, ya que es cuando ocurre la transición entre la infancia y la vida adulta. Ocurren muchos conflictos, pues al mismo tiempo que no son vistos como niños dependientes, tampoco son considerados lo suficientemente adultos para tomar las propias decisiones y ser dueños de sus vidas. La adolescencia es una etapa natural del desarrollo, teniendo un carácter universal y abstracto. Inherente al desarrollo humano, la adolescencia es percibida como una fase difícil, prácticamente semipatológica, que se presenta cargada de conflictos "naturales" fundamental en lo que se refiere a la salud, pues la transición de niño a adolescente y de este a adulto joven tiene importantes implicaciones en el desarrollo de muchos problemas de salud y en el desarrollo de cuidados de salud ${ }^{(2)}$.

En cuanto a la fase de adultos jóvenes, esta es definida como de los 19 a los 24 años. Periodo este en el cual suceden importantes cambios y se da el continuo desarrollo humano de forma más perfecta que en la adolescencia, debido a la mayor edad y al aumento en cargos por parte de la sociedad y de sí mismo. Es en esta época de la vida que la mayoría de las personas forman relaciones que durarán para el resto de la vida, basadas en amistad, amor o sexualidad. Los jóvenes adultos, generalmente, están en el auge de su fuerza, energía y resistencia. La mayoría de los sentidos están más perfeccionados en el inicio de la vida adulta y las condiciones de salud tienden a ser favorables ${ }^{(3)}$.

En cuanto a las enfermedades crónicas, estas acompañarán a sus portadores el resto de su vida, ya que la persona vivenciará cambios en su modo de vivir, en su rutina y en su relación con el medio. Individuos que se descubren portadores de cambios crónicos están sometidos, todo el tiempo, a situaciones nuevas y teniendo que 
adaptarse a las mismas. Factores presentes en la vida de portadores de enfermedad crónica: uso regular de medicación, alguna con potencialidad para el desarrollo de efectos colaterales; visitas constantes a médicos, otros profesionales e instituciones de salud; dificultad para practicar deportes; convivencia diaria con el dolor y/o el malestar; programas terapéuticos extensos, complejos y que imponen limitaciones; necesidad de cuidados continuos y cambios en la rutina ${ }^{(4-5)}$. Es posible que pensamientos negativos rodeen, esporádicamente, la vida del portador de una enfermedad crónica cuando hubiera cualquier complicación o cuando el mismo se sienta inseguro en relación a su estado. Según algunos autores ${ }^{(6)}$, el desarrollo de enfermedades crónicas está asociado al deterioro, a la reducción de competencias, al aumento de la necesidad de ayuda, al dolor físico y emocional resultante en pérdida de la independencia y al aumento de la necesidad de asistencia.

Apoyo social es cuando un grupo de personas, sea este de cualquier círculo de convivencia del individuo, ayuda a este, de alguna forma, a encarar determinada situación que acontezca en su vida. Puede ser definido como funciones desempeñadas por el grupo para favorecer al individuo en las situaciones de vida, pudiendo ser familiares, amigos, vecinos y otros. Al conjunto por ellos formado se le llama red de relaciones sociales. Desde esta perspectiva, la mayoría de los estudios desarrollados destaca, en el análisis del apoyo social, la percepción que los individuos tienen sobre sí mismos, que representa la creencia generalizada de que son estimados, que los otros se interesan por ellos, que están disponibles cuando precisan y satisfechos con las relaciones que tienen ${ }^{(7-8)}$.

El apoyo social puerde ser entendido como la calidad del soporte emocional a partir de las relaciones establecidas en las redes sociales. Estudios comprueban que individuos con apoyo de la familia y amigos tienen mejores condiciones de salud física y mental, teniendo en cuenta los recursos emocionales ${ }^{(9)}$. Así, podemos considerar que el concepto de apoyo emocional enlaza directamente con el apoyo social, o sea, el apoyo socio-emocional. Apoyo este que ayuda a procurar los recursos emocionales y prácticos que el individuo precisa. El afecto, la asistencia y la información de los amigos y familiares y las relaciones de compañerismo ejercen un efecto de protección y bienestar ${ }^{(19)}$.

De este modo, cuando relacionamos todas las definiciones anteriores, podemos entender la importancia del apoyo socio-emocional tanto para el adolescente como para el adulto joven portador de una enfermedad crónica. La familia y el portador de enfermedad crónica merecen atención especial, no solamente desde el punto de vista biológico, sino también en las dimensiones psicológicas, sociales, económicas y espirituales $^{(10)}$.

La adolescencia es definida como una fase de dificultades, transiciones y descubrimientos que, incluso sin la presencia de enfermedad alguna, ya sería importante el apoyo socio-emocional de los individuos que lo rodean. Un gran número de estudios enfatiza la importancia del apoyo socio-emocional durante el desarrollo en la adolescencia, tanto en relación con el bienestar físico como psicológico ${ }^{(7-8)}$. Visto lo que fue citado anteriormente, podemos vislumbrar lo esencial que es el apoyo socioemocional en la adolescencia cuando esta se une a la enfermedad crónica, pudiendo esta fase volverse aún más dificultuosa para el sujeto. La enfermedad crónica impone modificaciones en la vida del adolescente y su familia, exigiendo readaptaciones frente a la nueva situación y estrategias para su enfrentamiento. Este proceso de adaptación y aceptación depende de la complejidad y gravedad de la enfermedad, de 
la fase en que ellos se encuentran y de las estructuras disponibles para satisfacer sus necesidades y readquirir el equilibrio ${ }^{(11)}$.

En la fase considerada como de adulto joven, durante la definición, cuando es mencionado el hecho de que están en el "auge de su fuerza, energía y resistencia y que las condiciones de salud tienden a ser más favorables", ya ocurren citaciones que no "combinan" con la aparición de dolencias, principalmente enfermedades crónicas, que tienen carácter definitivo. Tal definición nos lleva a creer que la aparición de la enfermedad tenderá a traer problemas que necesitarán de apoyo socio-emocional para ser enfrentados. Las relaciones sociales afectan positivamente los sistemas biológicos, los comportamientos de salud y el bienestar psicológico de los individuos. Así, el involucrar a otras personas (familia, amigos, profesionales de salud, líderes religiosos) en el cuidado y en el apoyo para el manejo de consecuencias adversas de la enfermedad puede favorecer la adaptación y el ajuste del paciente a la enfermedad crónica ${ }^{(12)}$.

Así, este estudio tiene como objetivos: Identificar la importancia del apoyo socioemocional en adolescentes y adultos jóvenes portadores de enfermedad crónica y la importancia del equipo de salud, en especial el enfermero, en dar apoyo socioemocional.

\section{METODOLOGÍA}

Se trata de una investigación cualitativa y bibliográfica según determinado autor ${ }^{(13)}$, "[...] se realiza a partir del registro disponible, derivado de estudios anteriores en documentos impresos, como libros artículos, tesis, etc. Se sirve de datos o de categorías teóricas ya trabajadas por otros investigadores y debidamente registradas."

El levantamiento de datos se realizó a través de la busca de artículos en los periódicos del área, en las bases electrónicas de datos de la Biblioteca Virtual en Salud (BVS), SCIELO, LILACS e BDENF, usando los descriptores: apoyo social, adulto joven, adolescente y enfermedad crónica. Inicialmente, se investigó con los descriptores aislados y después asociados en parejas y tríos. La selección de los artículos se realizó a través de la lectura de los mismos, evaluando cuáles tenían mayor relación con el objeto de esta investigación. La mayoría de los artículos que fueron descartados estaban relacionados con la gravidez en la adolescencia y con el consumo de drogas en la juventud. De esta forma, se seleccionaron 13 artículos que contenián asuntos relacionados con el tema de la investigación y que colaborarían para el cumplimiento de los objetivos de la misma. Los descriptores se usaron individualmente y asociados, siendo la forma como fueron asociados y el número de artículos encontrados en cada descriptor, inclusive en las asociaciones, distribuídos en el cuadro 1 y divididos por las bases de datos utilizadas. De todos los artículos seleccionados, usamos el periodo de 2000 a 2010 como criterio de inclusión, así como la relación directa con el tema y los objetivos de la presente investigación. 
Cuadro 1. Artículos encontrados en cada descriptor en las bases de datos. Niterói, 2010.

\begin{tabular}{|l|l|l|l|}
\hline & SCIELO & LILACS & BDENF \\
\hline Apoyo Social & 66 & 554 & 46 \\
\hline Enfermedad crónica & 116 & 2871 & 129 \\
\hline Adulto Jovem & 2 & 1289 & - \\
\hline Adolescente & 1514 & 50587 & 1072 \\
\hline Apoyo Social + Adolescente + Enfermedad crónica & - & 2 & - \\
\hline Apoyo Social + Enfermedad crónica & 1 & 7 & - \\
\hline Apoyo social + Adolescente & 2 & 112 & 4 \\
\hline Apoyo Social + Adulto Joven & - & 5 & - \\
\hline Apoyo social + Adulto Joven + Enfermedad crónica & - & - & - \\
\hline Adulto Joven + Enfermedad crónica & - & 34 & - \\
\hline Adolescente + Enfermedad crónica & 2 & 479 & 13 \\
\hline
\end{tabular}

A partir de la lectura sistemática de los artículos, estos se dividieron en diferentes métodos y abordajes, distribuídos en el cuadro 2.

Los métodos y los abordajes a ser divididos son:
a- Investigación exploratoria/descriptiva de campo
b- Relato de experiencia
c- Investigación bibliográfica
d- Investigación comparativa
A- Abordaje cualitativo
B- Abordaje cuantitativo
C- Abordaje cuanti-cualitativo

Cuadro 2. Demostrativo de los métodos utilizados en los artículos encontrados. Niterói, 2010.

\begin{tabular}{|l|l|l|l|l|l|l|}
\hline \multicolumn{4}{|l|}{ MÉTODO } & \multicolumn{4}{l|}{ ABORDAJE } \\
\hline A & b & c & d & A & B & C \\
\hline 11 & - & 1 & 1 & 5 & 2 & 6 \\
artículos & & artículo & artículo & artículos & artículos & artículos \\
\hline
\end{tabular}

\section{RESULTADOS Y DISCUSIÓN}

\section{Importancia del apoyo socio-emocional en adolescentes y adultos jóvenes portadores de enfermedad crónica}

La presente categoría tiende a a relatar la importancia que hay en proporcionar apoyo socio-emocional a los adolescentes y adultos jóvenes portadores de enfermedad crónica, ya que con el referido apoyo se hace más fácil relacionarse con la enfermedad en el día a día y tener esperanzas de calidad de vida incluso en la condición de enfermos crônicos.

Algunas hipótesis intentan explicar los efectos del apoyo socio-emocional en la salud y en las enfermedades: (1) la hipótesis de que el apoyo socio-emocional tiene efectos 
a nivel de las respuestas neuroendocrinas, disminuyendo la ansiedad y, luego, la tensión muscular; (2) tiene efectos a nivel de la autoestima, aumentándola; (3) tiene efectos en la depresión, disminuyéndola y llevando a las personas a evaluar los estresores como menos graves, lo que podrá disminuir la ansiedad y aumentar la capacidad de la persona para lidiar con las situaciones estresantes ${ }^{(14-15)}$. De este modo, podemos percibir cómo el apoyo socio-emocional puede ser considerado benéfico en personas que se encuentran con algún tipo de enfermedad, pues se hace mucho más difícil enfrentar la situación sin la ayuda y amparo debidos de personas que son consideradas de confianza y que poseen una relación afectiva con el enfermo(a).

En el caso de la enfermedad crónica, este apoyo es aún más importante debido al estrés que las modificaciones de la cronicidad de la enfermedad causan en la vida de su portador, sea cual sea su edad. Es preciso considerar la vulnerabilidad del enfermo crónico y el sentimiento de desamparo en la situación del tratamiento. Es en este punto que la satisfacción con el apoyo social importa. Es la amplitud de la red que proporciona mayor seguridad y, por tanto, satisfacción del enfermo, dado que él cuenta con más recursos, incluso aun cuando no sepa correctamente la dimensión de su importancia ${ }^{(9)}$. Luego, por más que el enfermo no tenga noción de cuan importante es tener una red de personas a su alrededor que le proporcione apoyo frente a la situación que está enfrentando, incluso así esa red traerá beneficios respecto al tratamiento y enfrentamiento de la enfermedad, pues funcionará como un "puerto seguro" en los momentos de flaqueza, que posiblemente puedan ocurrir en el curso de la enfermedad crónica. El apoyo de los amigos, de un cuidador o de la familia parece asociarse al aumento del bienestar, a la prevención del estrés, así como a menor soledad, menor vulnerabilidad y mayor seguridad, ya que vivir solo parece tener un efecto negativo en la satisfacción con los domínios físico, social y psicológico de la vida ${ }^{(16-17)}$.

En lo que se refiere a los adolescentes y adultos jóvenes, estos factores estresantes pueden aumentar, ya que pasan por fases, tanto uno como otro, en que no se espera caer enfermo, pues es cuando se considera que el individuo está en la mejor fase de la vida, con buena salud y energía, en el auge de su fuerza física y de su vigor. De este modo, encontrarse en una situación de enfermedad, aún más una enfermedad incurable, puede ser de hecho una situación que generará muchas dificultades en la vida de estas personas.

En relación a la adolescencia, dado que es una fase de formación de identidad, descubrimientos sexuales, gran importancia a la apariencia física y creación de independencia, este tipo de situación puede, particularmente, forjar situaciones en las que el adolescente precisa sentirse apoyado, tanto por la familia como por los amigos, pues de esa forma él no se sentirá excluido, lo que, directamente, ayudará en el seguimiento del tratamiento adecuado. Se verificó también que el impacto relacionado con el autocuidado y bienestar en adolescentes con enfermedad crónica es mucho mayor en aquellos que recibían apoyo socio-emocional, tanto de la familia como de los amigos ${ }^{(18)}$.

En la fase del adulto joven es difícil lidiar con la enfermedad, pues es cuando se forman los vínculos más fuertes relacionados con la vida afectiva, ocurre la selección y configuración en la vida académica y profesional, o sea, en medio de tantas definiciones que ocurren, acontece también que el descubrimiento de una enfermedad definitiva puede generar un estrés grande en la mente de esa persona. La presencia 
de una enfermedad crónica puede ser una experiencia difícil, visto que su diagnóstico frecuentemente viene acompañado de considerable estrés y miedo. En esa perspectiva, el apoyo social debe ser incluido en el plan de cuidados, para que el adulto joven pueda enfrentar positivamente las demandas impuestas por la enfermedad $^{(19)}$. La intervención dirigida al suministro del apoyo socio-emocional adecuado, desarrollada tanto con personas afectadas por enfermedades crónicas como con sus círculos de convivencia, amigos y familia, puede contribuir a la mejora de las condiciones de salud de los pacientes, ya que tiende a aumentar la adhesión al tratamiento y su compromiso en comportamientos de salud ${ }^{(20)}$.

Por tanto, de acuerdo con la literatura utilizada, el descubrimiento y la convivencia con una enfermedad crónica en ambas $s$ fases puede considerarse un gran estrés, ya que todas las dificultades relacionadas con la enfermedad se unirán a las dificultades encontradas en la fase en que se está viviendo. De este modo, podemos concluir que el apoyo socio-emocional, sea familiar, de amigos o profesionales de salud, se torna prácticamente fundamental en el auxilio de estos jóvenes, para que tengan el comportamiento más adecuado posible dentro de la enfermedad que los acompaña, para que haya un buen enfrentamiento de la enfermedad y para que puedan considerar que la calidad de vida no es imposible, incluso estando presente la enfermedad.

\section{Apoyo socio-emocional proporcionado por el equipo de salud}

El equipo de salud, de un modo general, tiende a mirar mucho el aspecto fisiológico de la enfermedad, dando más atención al cuidado de las señales y síntomas físicos sin importar el aspecto social, el cómo la enfermedad puede afectar la vida de los jóvenes, perjudicando las relaciones con amigos, familia y otros círculos de convivencia, pudiendo generar depresión, estrés, miedo e inseguridad, causando así una mala convivencia de la enfermedad, generando baja adhesión al tratamiento y actitudes que pueden ser perjudiciales para que este vaya bien. De este modo, se enfatiza que hoy es necesario que exista un proceso de cambio en el equipo de salud, que antes se volvía más para los procedimientos técnicos. Ahora, cada vez más, se muestra la necesidad de intentar promover un cuidado holístico, o sea, un mirar físico, psicológico y social tanto sobre el cliente como sobre su familia ${ }^{(21)}$. El adolescente y el adulto joven portadores de enfermedad crónica merecen atención especial, no solamente desde el punto de vista biológico y técnico, sino también en las dimensiones psicológicas, sociales, económicas y espirituales. Además de esto, es importante estar de acuerdo con que el soporte social ayuda a la terapéutica juntamente con los amigos y el hospital ${ }^{(9)}$.

Es importante saber que el equipo de salud, principalmente el enfermero, es considerado uno de los medios de apoyo socio-emocional para los adolescentes y adultos jóvenes, ya que se encuentra en permanente contacto con ellos. De este modo, se enfatiza el papel del enfermero como principal enlace entre el equipo de salud y las redes de apoyo, pues este es capaz de establecer una relación de proximidad con las personas y sus familias, además de encontrarse en una posición privilegiada para promover recursos de apoyo disponibles a las personas, por medio de intervenciones tanto en la red social como en el equipo de salud ${ }^{(22)}$.

Sabiendo de la importancia del apoyo socio-emocional procurado por el profesional de salud, es necesario que tengamos la noción de cuan importante es proporcionar siempre un ambiente confortable y acogedor al paciente y mantener siempre una 
relación de confianza y respeto con él, pues muchas veces, dependiendo de cada persona, aquel ambiente con aquellos profesionales es el único lugar en el cual podrá recibir apoyo. La enfermedad crónica debilita al portador y familia, siendo esencial que el servicio de salud frecuentado por ellos sea acogedor y agradable y, también, un lugar donde él pueda dividir angustias, incertidumbres y miedos sin ser reprimido, prestando así una atención humanizada e individualizada ${ }^{(21)}$.

Tratándose de adolescentes y adultos jóvenes, dado que son fases de la vida difíciles y que pueden generar estrés en ellos, aumenta la necesidad de realizar una atención adecuada, humanizada e individualizada, procurando entender sus miedos y angustias, pues en estos casos, si no se ha creado una relación de confianza en la cual el profesional deje clara la intención de apoyo, no habrá comunicación adecuada y, consecuentemente, la atención no ocurrirá de la mejor forma posible. El sentido común nos lleva a pensar que un adolescente o adulto joven con una enfermedad para toda la vida percibirá que tiene una mayor posibilidad de calidad de vida si siente que tiene apoyo y que puede contar con él ${ }^{(23-24)}$.

Debemos considerar que el adolescente y el adulto joven vivencian sentimientos y situaciones complejas en el cotidiano de la enfermedad crónica, lo que determina la importancia de los profesionales de salud en conocer estas demandas e incorporarlas al plan de cuidados ${ }^{(10)}$. Cuando se da la incorporacióno de estos tipos de demandas al plan de cuidados, una de las formas que podemos ejercer, como profesionales de salud, es el apoyo socio-emocional, pues es a partir de este que podremos proporcionar una posible "descomplejidad" de los sentimientos y situaciones que los acompañan y, consecuentemente, estos podrán realizar el tratamiento propuesto de la mejor forma posible, sin que haya complicaciones para sus condiciones, ya que la intervención dirigida al suministro de apoyo adecuado, desarrollada también por los cuidadores de personas afectadas por enfermedades crónicas, puede contribuir a la mejora de las condiciones de salud de los pacientes, una vez que tiende a aumentar la adhesión al tratamiento y su compromiso en comportamientos saludables ${ }^{(20)}$.

\section{CONCLUSIÓN}

Podemos llegar a la conclusión de que el apoyo socio-emocional es de fundamental importancia en el cuidado de pacientes portadores de cualquier tipo de enfermedad. El hecho de ser una enfermedad crónica solo incrementa la situación, dejando al individuo aún más vulnerable por el hecho de ser una enfermedad incurable, que traerá estrés diario a su vida y, por tanto, convirtiendo a este individuo en alguien necesitado de cuidados técnicos y biológicos y de apoyo socio-emocional tanto de los amigos y familiares como del equipo de salud que trabaja junto al mismo.

En relación a la presencia de enfermedades crónicas en adolescentes y adultos jóvenes, podemos concluir que es un hecho aún más preocupante, ya que ambos se encuentran en fases que son consideradas problemáticas: la adolescencia por ser un periodo de transición y construcción de la identidad y la fase de adulto joven por el hecho de ser un periodo donde ocurren las opciones relacionadas con la estabilidad profesional, conyugal y con la afirmación de la identidad que fue adquirida en la adolescencia. Ya que ambas fases son consideradas fases de buena salud y extrema vitalidad, la aparición de una enfermedad, principalmente una que no tenga cura, puede traer miedos, angustias, ansiedad y comportamientos de rebeldía en relación al tratamiento y la buena convivencia con la enfermedad. De este modo, podemos notar que el apoyo socio-emocional, sea familiar, de amigos, religión, profesionales de 
salud o cualquier otro, se torna fundamental para que estos pacientes tengan una buena adhesión al tratamiento y consigan convivir con la enfermedad de la forma más adecuada posible y menos perjudicial para el tratamiento.

En lo que se refiere al apoyo socio-emocional relacionado exclusivamente con el profesional de salud, vemos que tiene una importancia fundamental. Es un acto de cuidado que debe ser más explorado por los profesionales de un modo general, inclusive incluyendo actividades relacionadas con la promoción del apoyo social dentro del plan de cuidados de los adolescentes y de los adultos jóvenes, así como de los demás pacientes, ya que puede ser un factor que ayudará en el tratamiento, como ya fue citado anteriormente.

Es importante destacar la deficiencia en relación con los artículos relacionados con el adulto joven, en especial cuando está relacionado con la dolencia crónica o apoyo social, ya que observando los números de artículos encontrados en todas las bases de datos utilizadas, podemos notar una gran discrepancia en relación a los demás descriptores. El descriptor "adulto joven" cuando utilizado solo, sobre todo cuando está asociado a los demás descriptores utilizados como "adulto joven + enfermedad crónica", "adulto joven + apoyo social" y "adulto joven + enfermedad crónica + apoyo social", prácticamente ningún artículo relacionado con el tema fue encontrado en las tres bases de datos utilizadas.

\section{REFERENCIAS BIBLIOGRÁFICAS}

1. Bock, AMB, A adolescência como construção social: estudo sobre livros destinados a pais e educadores. Psicol. Esc. Educ. (Impr.), [periódico na internet] 2007 Jan.-Jun. [acessado 2010 Jun. 20]; 11(1): [cerca de 13 p.] Disponível em: http://www.scielo.br/pdf/pee/v11n1/v11n1a07.pdf

2. Willians, P, Hombeck, G, Greenley, R, Adolescent Health Psychology In. Almagiá, EB, Cerna, R, Kramm, D, Véliz, V, Problemas de salud, estrés, afrontamiento, depresión y apoyo social en adolescentes. Ter Psicol, [periódico na internet] 2006 Jun. [acessado 2010 Jul. 13]; 24(01), [cerca de 6 p.] Disponível em: http://redalyc.uaemex.mx/pdf/785/78524106.pdf

3. Papalia, DE, Olds, SW, Desenvolvimento Humano In. Melo, DM, Breves considerações sobre a vida do jovem adulto Acessa.com, [página na internet] 2004, 19 Mar. Disponível em: http://www.acessa.com/viver/arquivo/psique/2004/03/19jovem/

4. McQuaid, EL, \& Walders, N Pediatric asthma. In. Salomão Jr. JB, Miyasaki, MCOS, Cordeiro, JA, Domingos, NAM, Valério, NI Asma, competência social e transtornos comportamentais em crianças e adolescentes. Estud Psicol. (Campinas), [periódico na internet] 2008 Abr.-Jun. [acessado em 2010 Jul. 11]; 25(02), [cerca de 7 p.] Disponível em: http://www.scielo.br/pdf/estpsi/v25n2/a03v25n2.pdf

5. Miyazaki, MCOS, Risso, KR, Salomão, JB Características e tratamento da asma na infância. In. Salomão Jr. JB, Miyasaki, MCOS, Cordeiro, JA, Domingos, NAM, Valério, $\mathrm{NI}$ Asma, competência social e transtornos comportamentais em crianças e adolescentes. Estud Psicol. (Campinas), [periódico na internet] 2008 Abr.-Jun. [acessado em 2010 Jul. 11]; 25(02), [cerca de 7 p.] Disponível em: http://www.scielo.br/pdf/estpsi/v25n2/a03v25n2.pdf

6. Gignac, MA, Cott, CA Conceptual model of independence and dependence for adults with chronic physical illness and disability. In. Resende, MC, Santos, FA, Souza, MM, Marques, TP, Atendimento psicológico a pacientes com insuficiência renal crônica: em busca de ajustamento psicológico. Psicol Clin., [periódico na 
internet] 2007 Dez. [acessado em 2010 Jun.03]; 19(2), [cerca de 11p.] Disponível em: http://www.scielo.br/scielo.php?script=sci_arttext\&pid=S0103-

$56652007000200007 \&$ Ing $=$ en\&nrm $=$ iso

7. Heller, K, Swindle, R, Dusenbury, L Component social support processes: Comments and integration. In. Antunes, C, Fontaine, AM, Percepção de apoio social na adolescência: análise fatorial confirmatória da escala social support appraisals. Paidéia, [periódico na internet] 2005 [acessado em 2010 Abr. 14]; 15(32), [cerca de 11p.] Disponível em: http://www.scielo.br/pdf/paideia/v15n32/05.pdf

8. Vaux, A Social support: Theory, research and interventions. In. Antunes, C, Fontaine, AM Percepção de apoio social na adolescência: análise fatorial confirmatória da escala social support appraisals. Paidéia, [periódico na internet] 2005 [acessado em 2010 Abr. 14]; 15(32), [cerca de 11p.] Disponível em: http://www.scielo.br/pdf/paideia/v15n32/05.pdf

9. Rudnicki, T Preditores de qualidade de vida em pacientes renais crônicos, Estud Psicol. (Campinas), [periódico na internet] 2007 [acessado em 2010 Abr. 14]; 24(03), [cerca de 8p.] Disponível em: http://www.scielo.br/pdf/estpsi/v24n3/a06v24n3.pdf

10. Beltrão, MRLR, Vasconcelos, MGL, Pontes, CM, Albuquerque, MC Câncer infantil: percepções maternas e estratégias de enfrentamento frente ao diagnóstico. $J$. Pediatr. (Rio J.), [periódico na internet] 2007 [acessado em 2010 Jul. 20]; 83(06), [cerca de 4p.] Disponível em: http://www.scielo.br/pdf/jped/v83n6/v83n6a14.pdf

11. Vieira, MA, Lima, RAG Crianças e adolescentes com doença crônica: convivendo com mudanças. Rev. Latino-Am. Enfermagem, [periódico na internet] 2002 [acessado em 2010 Abr. 14]; 10(4), [cerca de 8p.] Disponível em: http://www.scielo.br/pdf/rlae/v10n4/13368.pdf

12. Martire, ML, Schulz, $R$ Involving family in psychosocial interventions for chronic illness. In. Rodrigues, MA, Seidl, EMF A importância do apoio social em pacientes coronarianos. Paidéia, [periódico na internet] 2008 [acessado em 2010 Ago. 24]; 18(40), [cerca de 8p.] Disponível em: http://www.scielo.br/pdf/paideia/v18n40/06.pdf 13. Severino, AJ Metodologia do Trabalho Científico. Editora Cortez, São Paulo, 2007 14. Seeman, TE, McEwen, BS Impact of social environment characteristics on neuroendocrine regulation. In. Silva, I, Pais-Ribeiro, J, Cardoso, H, Ramos, H, Carvalhosa, SF, Dias, S, Gonçalves, A Efeitos do apoio social na qualidade de vida, controle metabólico e desenvolvimento de complicações crônicas em indivíduos com diabetes, Psicol. saúde doenças., [periódico na internet] 2003 [acessado em 2010 Ago. 24]; 4(01), [cerca de 11p.] Disponível em: http://www.scielo.oces.mctes.pt/pdf/psd/v4n1/v4n1a02.pdf

15. Wills, TA Social support and health. In. Silva, I, Pais-Ribeiro, J, Cardoso, H, Ramos, H, Carvalhosa, SF, Dias, S, Gonçalves, A Efeitos do apoio social na qualidade de vida, controle metabólico e desenvolvimento de complicações Crônicas em indivíduos com diabetes, Psicol. saúde doenças., [periódico na internet] 2003 [acessado em 2010 Ago. 24]; 4(01), [cerca de 11p.] Disponível em: http://www.scielo.oces.mctes.pt/pdf/psd/v4n1/v4n1a02.pdf

16. Wikby, A, Hörnquist, JO, Stenström, U, Anderson, PO Background factors, longterm complications, quality of life and metabolic control in insulin dependent diabetes. In. Silva, I, Pais-Ribeiro, J, Cardoso, H, Ramos, H, Carvalhosa, SF, Dias, S, Gonçalves, A. Efeitos do apoio social na qualidade de vida, controle metabólico e desenvolvimento de complicações crônicas em indivíduos com diabetes, Psicol. saúde doenças., [periódico na internet] 2003 [acessado em 2010 Ago. 24]; 4(01), [cerca de 11p.] Disponível em: http://www.scielo.oces.mctes.pt/pdf/psd/v4n1/v4n1a02.pdf

17. Hanestad, BR Self-reported quality of life and the effect of different clinical and demographic characteristics in people with type 1 diabetes. In. Silva, I, Pais-Ribeiro, J, 
Cardoso, H, Ramos, H, Carvalhosa, SF, Dias, S, Gonçalves, A. Efeitos do apoio social na qualidade de vida, controle metabólico e desenvolvimento de complicações crônicas em indivíduos com diabetes, Psicol. saúde doenças., [periódico na internet] 2003 [acessado em 2010 Ago. 24]; 4(01), [cerca de 11p.] Disponível em: http://www.scielo.oces.mctes.pt/pdf/psd/v4n1/v4n1a02.pdf

18. Skinner, T, John, M, Hampson, S Social support and personal models of diabetes as predictors of self-care and well-being: A longitudinal study of adolescents with diabetes. In. Monje, MJA, Almagiá, EB Autoeficacia, Apoyo Social y Calidad de Vida en Adolescentes con Enfermedades Crónicas, Ter Psicol, [periódico na internet] 2008 [acessado em 2010 Jul. 21]; 26(02), [cerca de 8p.] Disponível em: http://redalyc.uaemex.mx/pdf/785/78511540002.pdf

19. Gomes-Villas Boas LC, Santos CB, Foss-Freitas MC, Pace AE A relação entre o apoio social e as características sociodemográficas das pessoas com diabetes mellitus. Rev. Gaúcha Enferm. [periódico na internet] 2009 [acessado em 2010 Abr. 24]; 30(3), [cerca de 6p.] Disponível em: http://www.seer.ufrgs.br/index.php/RevistaGauchadeEnfermagem/article/view/8635/69 91

20. Rodrigues, MA, Seidl, EMF A importância do apoio social em pacientes coronarianos. Paidéia, [periódico na internet] 2008 [acessado em 2010 Jun. 18]; 18(40), [cerca de 9p.] Disponível em: http://www.scielo.br/pdf/paideia/v18n40/06.pdf 21. Schwartz, E, Muniz, RM, Burille, A, Zillmer, JGV, Silva, DA, Feijó, AM, Bueno, MEN As redes de apoio no enfrentamento da doença renal crônica. REME Rev. Min. Enferm., [periódico na internet] 2009 [acessado em 2010 Jun. 18]; 13(02), [cerca de 8p.] Disponível em: http://www.enf.ufmg.br/site_novo/modules/mastop_publish/files/files_4c0e49f32d824.p df

22. Hutchison, C Social support: factors to consider when designing studies that measure social support. In. Gomes-Villas Boas LC, Santos CB, Foss-Freitas MC, Pace $A E$ A relação entre o apoio social e as características sociodemográficas das pessoas com diabetes mellitus. Rev. Gaúcha Enferm. [periódico na internet] 2009 [acessado em 2010 Jun. 18]; 30(3), [cerca de 6p.] Disponível em: http://www.seer.ufrgs.br/index.php/RevistaGauchadeEnfermagem/article/view/8635/69 91

23. Ethgen, O, Vanparijs, P, Delhalle, S, Rosant, S, Bruyere, O, Reginster, J Social Support and health related quality of life in hip and knee osteoarthritis. In. Urzúa, A. Calidad de Vida y Factores Biopsicosociales en Patologías Médicas Crónicas. Ter Psicol, [periódico na internet] 2008 [acessado em 2010 Abr. 24]; 26(02), [cerca de 7p.] Disponível em: http://redalyc.uaemex.mx/pdf/785/78511540007.pdf

24. Wilson, I, Cleary, P. Linking clinical variables with health related quality of life. In. Urzúa, A. Calidad de Vida y Factores Biopsicosociales en Patologías Médicas Crónicas. Ter Psicol, [periódico na internet] 2008 [acessado em 2010 Abr. 24]; 26(02), [cerca de 7p.] Disponível em: http://redalyc.uaemex.mx/pdf/785/78511540007.pdf

ISSN 1695-6141

๑ COPYRIGHT Servicio de Publicaciones - Universidad de Murcia 